\title{
Role of glucose and CcpA in capsule expression and virulence of Streptococcus suis
}

\author{
Correspondence \\ R. Goethe \\ ralph.goethe@tiho-hannover.de
}

Received 25 October 2010

Revised 11 February 2011

Accepted 17 February 2011

\author{
J. Willenborg, ${ }^{1}$ M. Fulde, ${ }^{2}$ A. de Greeff, ${ }^{3}$ M. Rohde, ${ }^{2}$ H. E. Smith, ${ }^{3}$ \\ P. Valentin-Weigand ${ }^{1}$ and R. Goethe ${ }^{1}$
}

\author{
${ }^{1}$ Institute for Microbiology, University of Veterinary Medicine, Hannover, Germany \\ ${ }^{2}$ Department of Microbial Pathogenesis, Helmholtz Center for Infection Research, \\ Braunschweig, Germany \\ ${ }^{3}$ Animal Sciences Group (ASG), Wageningen UR, PO Box 65, 8200 AB Lelystad, The Netherlands
}

\section{INTRODUCTION}

Streptococcus suis is a Gram-positive, facultative anaerobic pathogen colonizing the upper respiratory tract, preferentially the tonsils of swine. It is endemic in nearly all pigproducing industries. Some strains are able to cross the epithelial barrier, causing bacteraemia and resulting in

Abbreviations: ADS, arginine deiminase system; ANOVA, analysis of variance; CCA, carbon catabolite activation; CCR, carbon catabolite repression; COG analysis, Clusters of Orthologous Groups analysis; GAS, group A streptococci; LRR fixation procedure, lysine-acetatebased formaldehyde/glutaraldehyde ruthenium red-osmium fixation procedure; PTS, phosphotransferase system; qRT-PCR, quantitative RT-PCR.

The microarray data discussed in this paper are available from ArrayExpress (http://www.ebi.ac.uk/arrayexpress) under accession number E-MEXP-2946.

Two supplementary figures and two supplementary tables are available with the online version of this paper. meningitis, arthritis, endocarditis and bronchopneumonia. As a zoonotic agent, S. suis can cause meningitis and sepsis in humans (Arends \& Zanen, 1988; Chanter et al., 1993; Clifton-Hadley \& Alexander, 1980; Rosenkranz et al., 2003). Among the 33 serotypes described in S. suis, serotype 2 strains seem to be the most virulent (Gottschalk et al., 2010). However, the mechanisms contributing to the virulence of $S$. suis in pigs and humans are only poorly understood.

The capsule of highly virulent $S$. suis serotype 2 strains has been proven to be a major virulence factor (Charland et al., 1998; Smith et al., 1999). It is proposed that during infection, S. suis downregulates capsule expression for increased adhesion to epithelial cells and upregulates it for protection against phagocytosis after entering the bloodstream (Gottschalk \& Segura, 2000). Besides the capsular polysaccharide, the opacity factor of $S$. suis (ofs) is the only proven virulence factor for pig infection so far (Baums et al., 2006). Other virulence-associated factors have been 
described, such as the surface-associated muramidasereleased protein ( $m r p)$, the extracellular factor (epf), the secreted haemolysin suilysin $(s l y)$, the cytosolic and surface-located enzyme enolase (eno), surface antigen one (sao), and the arginine deiminase system (ADS) (Baums \& Valentin-Weigand, 2009; Vecht et al., 1992; Wisselink et al., 2000).

In bacteria, the generation of energy by the uptake and consumption of environmental carbohydrates is tightly regulated. Thus, the availability of sugars which can be metabolized easily, such as glucose, activates sugar catabolism and suppresses other energy-providing mechanisms (Titgemeyer \& Hillen, 2002). These phenomena are called carbon catabolite activation (CCA) and carbon catabolite repression (CCR), respectively, and are crucial during bacterial growth in culture, a phenomenon known as growth phase regulation (Seshasayee et al., 2006). The catabolite control protein A (CcpA) is the major mediator of CCR, repressing gene expression in the presence of excess sugar during growth (Kietzman \& Caparon, 2010; Titgemeyer \& Hillen, 2002; Zomer et al., 2007). In addition, CcpA has recently been shown to be important for the virulence of a number of bacterial species. Bacterial growth, haemolysin production, biofilm formation and capsule expression have been shown to be influenced by CcpA depletion in other streptococci (Shelburne et al., 2008; Wen \& Burne, 2002). In the human pathogen Streptococcus pneumoniae, mutation in CcpA results in attenuated virulence in mouse infection models (Giammarinaro \& Paton, 2002; Iyer et al., 2005). On the other hand, $\operatorname{cp} A$ deficiency in Streptococcus pyogenes leads to both hypervirulence and hypovirulence, in the same set-up of experimental mouse infection (Kinkel \& McIver, 2008; Shelburne et al., 2008). The mechanisms contributing to this, however, are poorly understood, and nothing is known about the contribution of CcpA to the virulence of $S$. suis.

During infection, S. suis has to adapt to different host environments in terms of sugar availability, $\mathrm{pH}$ and temperature, for example. We have previously shown that the alternative energy-providing system of S. suis, the ADS, is temperature-induced and expressed on the streptococcal surface (Winterhoff et al., 2002). Furthermore, we were able to show that the ADS is inducible by arginine and $\mathrm{O}_{2}$ tension, is subject to CCR, and is important for bacterial survival (Benga et al., 2004; Gruening et al., 2006).

In the present study we demonstrate that expression of virulence features, including capsule under conditions of high glucose availability, depends on CcpA. We further show that CcpA depletion in S. suis results in a strongly attenuated phenotype that resembles that of non-encapsulated S. suis. As a consequence, CcpA-deficient S. suis displayed poor resistance to phagocytic killing, in contrast to the parental strain. Overall, our data provide evidence that glucose-mediated regulation contributes to the virulence of $S$. suis and indicate that CcpA-dependent capsule expression might be a major component of carbon catabolite-regulated virulence.

\section{METHODS}

Chemicals, bacterial strains and growth conditions. If not otherwise stated, all chemicals were purchased from Sigma. The highly virulent serotype 2 strain 10 , the capsule-deficient strain $10 \Delta c p s E F$ (Smith et al., 1999; Vecht et al., 1996), the suilysin-deficient

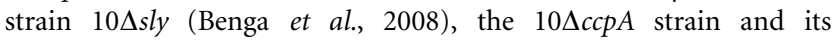
complementation $c 10 \Delta c c p A$ were used. Bacteria were routinely grown in Todd-Hewitt broth (THB; Becton Dickinson Diagnostics). Subculturing was performed overnight at $37{ }^{\circ} \mathrm{C}$ on Columbia Blood Agar Base (Difco) containing 6\% (v/v) sheep blood or horse blood supplemented with the appropriate antibiotics. To analyse bacterial growth, streptococci were grown in THB medium overnight and adjusted to $\mathrm{OD}_{600} 0.02$ on the next day. Then, $\mathrm{OD}_{600}$ was measured every hour and bacterial growth kinetics were determined in three independent experiments. For further experiments, bacteria were grown and harvested in different growth phases. As indicated in Fig. 2(a), time point $\mathrm{P}_{0}$ relates to early exponential, $\mathrm{P}_{1}$ to midexponential, $\mathrm{P}_{2}$ to late-exponential and $\mathrm{P}_{3}$ to early stationary bacterial growth. Escherichia coli was subcultured and maintained on LuriaBertani (LB) agar plates. If required, antibiotics were added at the following concentrations: spectinomycin at $100 \mu \mathrm{g} \mathrm{ml}^{-1}$ (S. suis) and $50 \mu \mathrm{g} \mathrm{ml}^{-1}$ (E. coli), erythromycin at $1 \mu \mathrm{g} \mathrm{ml}^{-1}$ (S. suis) and $400 \mu \mathrm{g}$ $\mathrm{ml}^{-1}$ (E. coli), ampicillin at $100 \mu \mathrm{g} \mathrm{ml}^{-1}$ (E. coli).

Glucose measurement. Determination of the glucose concentration in bacterial cultures was performed using the Glucose (GO) Assay kit (Sigma). Briefly, bacteria were grown in THB medium to the indicated time points (Fig. 2a) and then pelleted by centrifugation. The supernatants were collected and sterile-filtered (pore size $0.2 \mu \mathrm{m}$ ), and the glucose concentration (in $\mu \mathrm{g} \mathrm{ml}^{-1}$ ) was determined.

DNA techniques. Chromosomal $S$. suis DNA was prepared according to standard procedures as described by Sambrook et al. (1989). Isolation of streptococcal plasmid DNA was done with the Promega PureYield Midiprep system according to the manufacturer's instructions, with additional lysozyme $\left(10 \mathrm{mg} \mathrm{ml} \mathrm{ml}^{-1}\right)$ digestion. Plasmid preparations of $E$. coli were performed with the NucleoSpin Plasmid kit (Macherey-Nagel) according to the manufacturer's instructions. If not stated otherwise, all restriction enzymes were purchased from New England Biolabs (NEB). Southern analyses were performed with SnaBI-cleaved and SnaBI/NcoI double-digested genomic DNA according to standard protocols (Sambrook et al., 1989).

RNA isolation and Northern blotting. For total RNA extraction, the wild-type strain 10 and the respective mutant strain $10 \Delta c c p A$ were grown to time points $\mathrm{P}_{0}$ and $\mathrm{P}_{3}$ as described above. Harvested bacteria were then resuspended in $1 \mathrm{ml}$ TRIzol reagent (Invitrogen), ruptured using the FastPrep instrument (Qbiogene) three times for $45 \mathrm{~s}$ at intensity setting 6.5, and cooled on ice. After chloroform extraction and 2-propanol precipitation, the RNA was further purified using an RNeasy Mini kit (Qiagen) according to the manufacturer's recommendations. Northern blot analysis and hybridization were performed as described previously (Goethe \& Phi-van, 1998). The $c c p A-$ specific probe was amplified using the primer pair CcpA_for/ CcpA_rev (Supplementary Table S1).

Production and purification of recombinant CcpA. Recombinant CcpA was produced as a $6 \times$ His-tagged fusion protein in E. coli M15 with the QIAexpress $p \mathrm{QE}$ plasmid system (Qiagen). The respective ccpA gene was amplified by PCR from chromosomal DNA with 
(a)

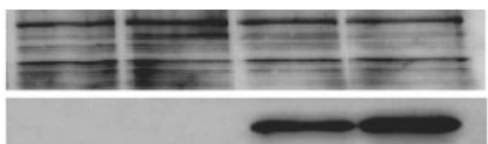

Silver stain
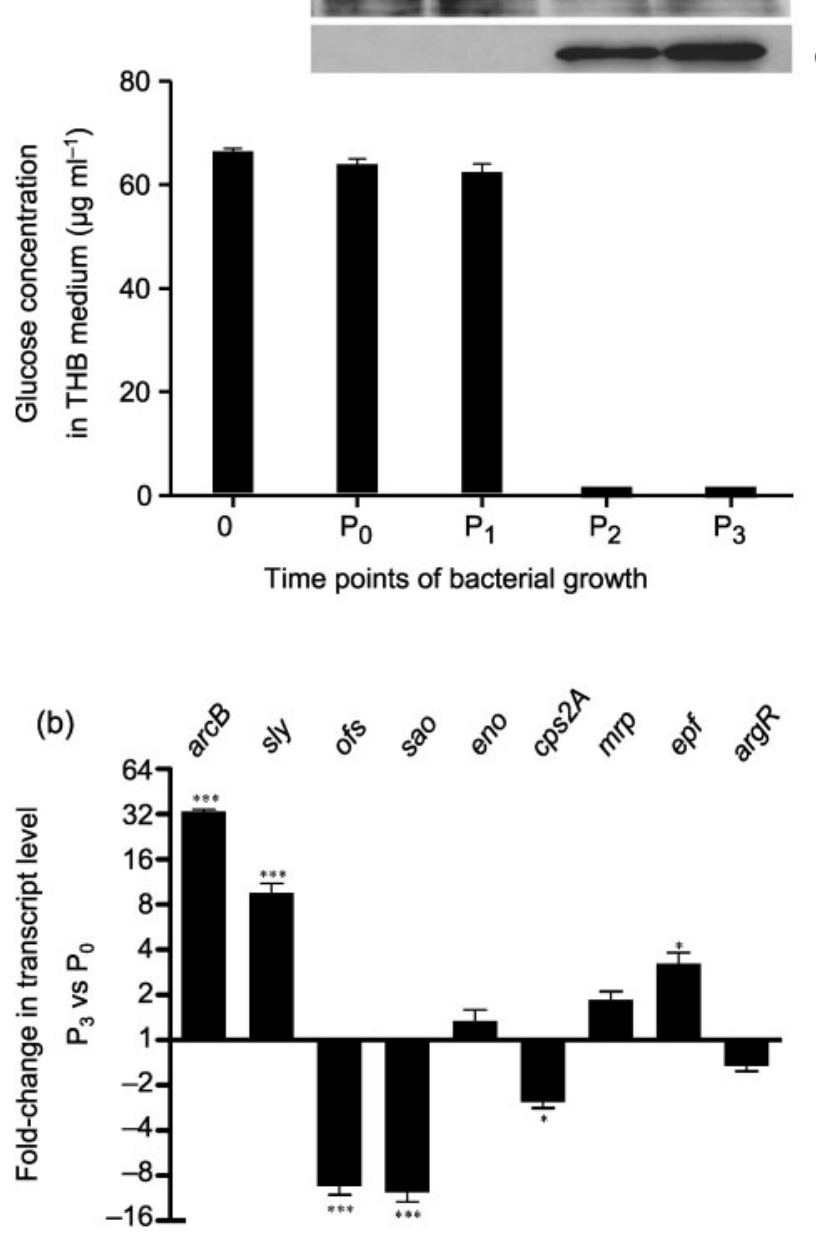

Fig. 1. Growth-dependent expression of virulence-associated factors. (a) Inset: immunoblot analysis with whole-cell lysates of $S$. suis wild-type strain 10 grown in THB medium. Time points $\mathrm{P}_{0}-\mathrm{P}_{3}$ reflect bacterial growth to early stationary phase, as depicted in Fig. 2(a). Five micrograms of bacterial cell lysate was separated by SDS-PAGE and blotted onto a PVDF membrane, and then probed with a polyclonal antiserum against ArcB. The detection of ArcB protein levels represents ArcABC expression (bottom panel). The silver-stained gel served as a loading control (top panel). The bar chart shows the quantification of glucose in the THB medium during bacterial growth of wild-type strain 10 . Ten millilitres of bacterial culture at the indicated time points (Fig. 2a) was cleared by centrifugation. Supernatants were sterile-filtered and the glucose concentration was determined as described in Methods. Results are given as mean \pm SD for three determinations. (b) Wild-type bacteria were grown in THB medium to the early exponential $\left(P_{0}\right)$ or early stationary $\left(P_{3}\right)$ growth phase, and relative transcript levels of the indicated genes were determined $\left(P_{3}\right.$ vs $\left.P_{0}\right)$. Data are mean \pm SD of three replicates performed in duplicate. Statistical analysis was performed by one-way ANOVA using Dunnett's adjustment to the non-regulated arg $R$ gene. Significance: ${ }^{\star} P<0.05,{ }^{\star \star} P<0.01$, ${ }^{\star \star \star} P<0.001$. Positive values indicate upregulation in stationary-grown bacteria. $\operatorname{arc} B$, ornithine carbamoyltransferase; sly, suilysin; ofs, opacity factor of S. suis; sao, surface-anchored protein; eno, enolase; $c p s 2 A$, capsule synthesis gene 2A; mrp, muramidase-released protein; epf, extracellular factor; $\arg R$, arginine repressor (SSU0588). the specific oligonucleotide primer pair CcpAfor_SacI and CcpArev_HindIII (Supplementary Table S1) using Pfu polymerase (Promega). The amplificate was digested with the respective enzymes and subsequently cloned into the SacI/HindIII-digested expression vector $p \mathrm{QE} 30$. The resulting plasmid $p \mathrm{QE3} 3$-rCcpA was introduced into E. coli strain M15 (Qiagen). Purified plasmid DNA was verified by restriction analysis and sequencing. After IPTG induction, CcpA was overexpressed as an N-terminal hexahistidyl derivative and isolated by $\mathrm{Ni}^{2+}$-nitrilotriacetic acid affinity chromatography according to the manufacturer's instructions (Macherey-Nagel, Protino Ni-TED 2000).

Polyclonal antiserum against purified recombinant CcpA was raised in a New Zealand white rabbit (Charles River Laboratories) by three consecutive immunizations with $100 \mu \mathrm{g}$ purified protein and $50 \%$ Freund's incomplete adjuvant. Generation of antibodies against ArcB was done as described previously (Gruening et al., 2006).

Mutagenesis and $\operatorname{ccp} \boldsymbol{A}$ complementation. The $\operatorname{ccp} A$ gene (SSU1202) was inactivated by insertion mutagenesis of S. suis strain
10. Briefly, the gene encoding CcpA was amplified from the streptococcal genome using primer pairs CcpAL/CcpALrev-HpaI and CcpAR/CcpARfor-HpaI (Supplementary Table S1). The PCR products were digested with $\mathrm{HpaI}$ and religated, resulting in a $311 \mathrm{bp}$ deletion of the native $c c p A$ gene. The ligation product was amplified using the primer pair CcpAL/CcpAR and cloned into the vector pGEM-T Easy (Promega). The resulting plasmid $p \mathrm{GEM}-c c p \mathrm{~A}$ was linearized by the restriction enzyme HpaI, and the PvuII-digested erythromycin-resistance cassette from $p \mathrm{IC}_{\text {erm }}$ was introduced to disrupt $c c p A$. Electroporation of $S$. suis was performed as previously described (Smith et al., 1995). Putative mutants were confirmed by Southern blot and Northern blot analysis (Supplementary Fig. S1).

For the complementation of the $c c p A$-deficient strain, the entire $c c p A$ gene and its putative promoter were amplified via a Pfu-based PCR using the primers CcpAfor_EcoRI and CcpArev_EcoRI. To obtain pGA14-CcpA, the amplified construct and the vector pGA14 were digested with EcoRI and ligated. The plasmid pGA14-CcpA was electrotransformed into competent $10 \triangle c c p A$ bacteria, and transformants were screened by plating onto spectinomycin-containing blood 


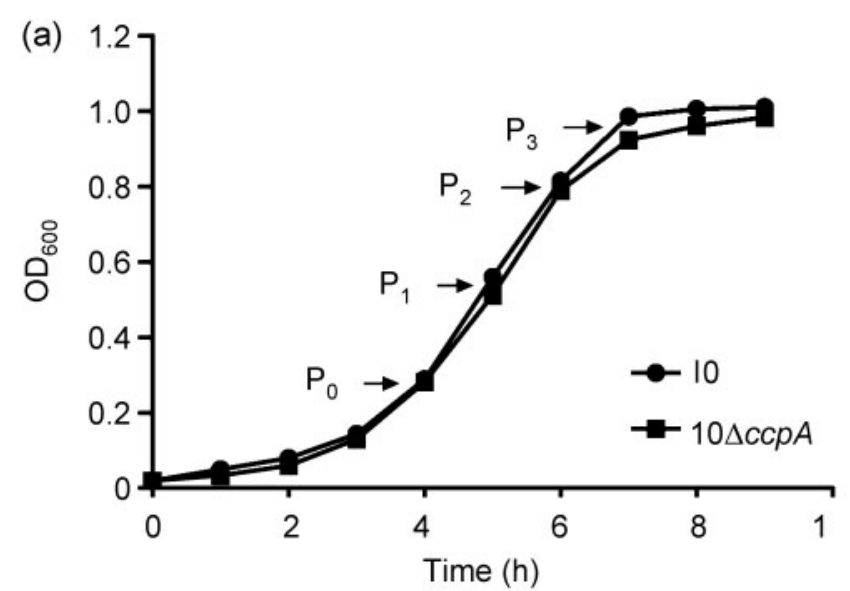

(b)
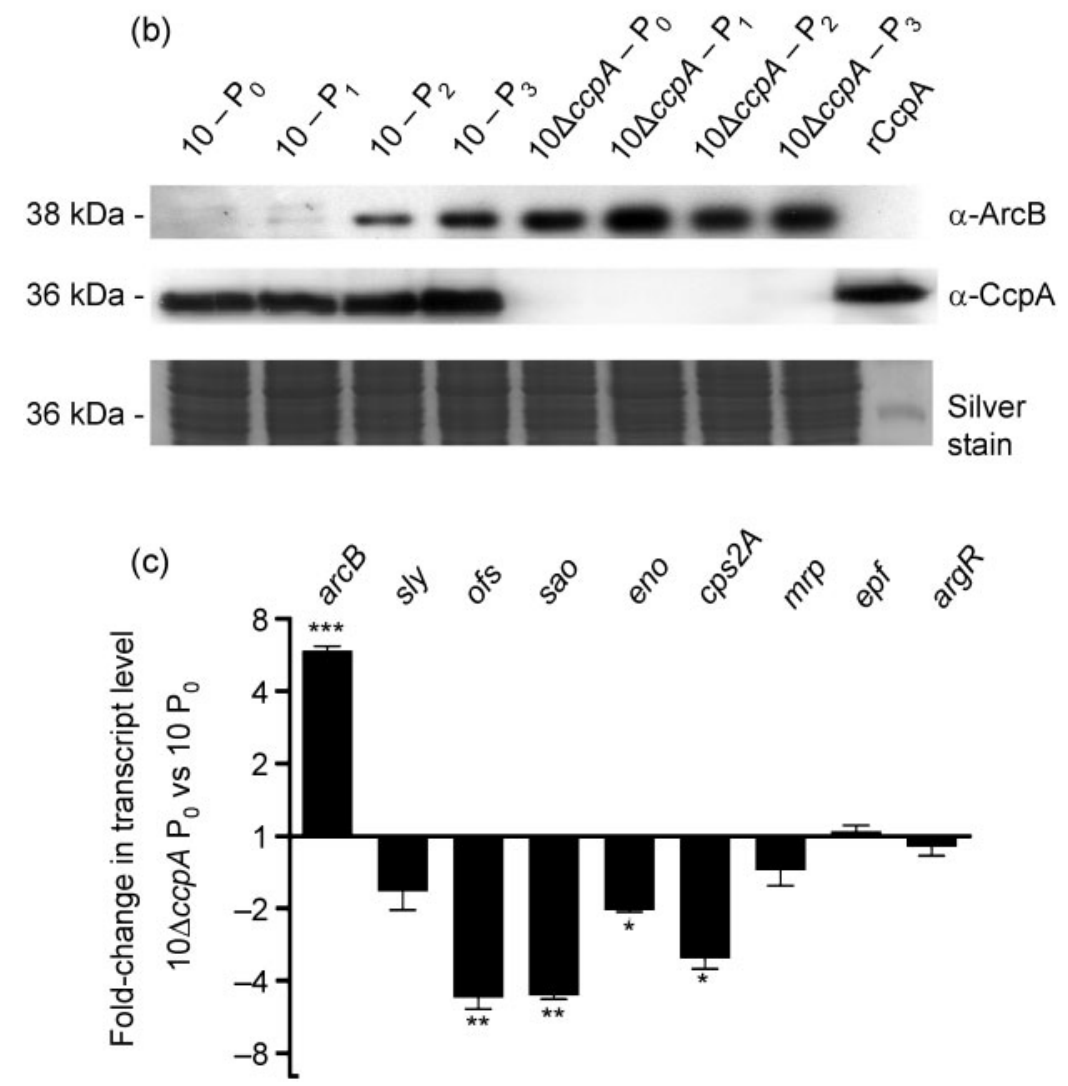

Fig. 2. Influence of $\mathrm{CcpA}$ deficiency on bacterial growth, arc $A B C$ operon expression and virulence-associated factor expression of S. suis. (a) $S$. suis wild-type strain 10 and strain $10 \triangle c c p A$ were cultured in THB medium as described in Methods. Growth was monitored at the indicated time points by determination of $\mathrm{OD}_{600}$. The graph shows the mean \pm SD of three independent experiments. (b) Immunoblot analysis with whole-cell lysates of $S$. suis wild-type strain 10 and strain $10 \triangle c c p A$ grown in THB to different time points $\left(P_{0}-P_{3}\right)$. Five micrograms of bacterial cell lysate was separated by SDS-PAGE and blotted onto a PVDF membrane, and then probed with a polyclonal antiserum against ArcB and CcpA. Recombinant CcpA (rCcpA) was included as a control, and the detection of ArcB protein levels represents ArcABC expression (top panel). The silver-stained gel served as a loading control (bottom panel). (c) Comparison of the relative transcript levels of the CcpA mutant strain $(10 \Delta c c p A)$ and wildtype strain 10 at early exponential growth $\left(P_{0}\right)$. qRT-PCR was performed and evaluated as described in the legend of Fig. 1(b). Data are mean $\pm S D$ of three replicates performed in duplicate. Statistical analysis was performed by one-way ANOVA using Dunnett's adjustment to the non-regulated argR gene. Significance: ${ }^{\star} P<0.05,{ }^{\star \star} P<0.01,{ }^{\star \star \star} P<0.001$. Positive values indicate upregulation in strain $10 \Delta c c p A$. See Fig. 1, legend, for an explanation of the genes shown. agar plates. Putative $c 10 \Delta c c p A$ transformants were confirmed by Western blot analysis using polyclonal anti $(\alpha)$-CcpA antiserum (Supplementary Fig. S1).

Preparation of whole-cell lysates and Western blot analysis. Ten millilitres of bacterial cultures representing the desired growth phase were harvested and centrifuged for $15 \mathrm{~min}$ at $4000 \mathrm{~g}$. The pellet was resuspended in $1 \mathrm{ml}$ cell lysis buffer [CLB; $50 \mathrm{mM}$ Tris/HCl (pH 8.0), 10 mM EDTA, $1 \%$ SDS, $1 \times 4$-(2-aminoethyl)benzenesulfonyl fluoride hydrochloride (AEBSF; Calbiochem)], and whole bacterial cell lysates were obtained by sonication (Branson, Cell Sonifier) and subsequent centrifugation. Protein concentrations were determined using the Bio-Rad $\mathrm{D}_{\mathrm{c}}$ Protein Assay. For CcpA Western blotting, proteins were separated by SDS-PAGE with a $4 \%$ stacking and a $12 \%$ separating gel. Samples were blotted onto a PVDF membrane (Serva), and membranes were blocked overnight with $3 \%$ BSA. Then, membranes were incubated with polyclonal antiserum raised against CcpA (diluted $1: 1000$ in 1\% BSA) for $60 \mathrm{~min}$. Membranes were developed with horseradish peroxidase-conjugated anti-rabbit IgG (Amersham) diluted 1:10000 in 1\% BSA and the SuperSignal West Pico Chemiluminescent Substrate (Pierce) as described by the manufacturer. Western blot analysis with $\alpha$-ArcB polyclonal serum was done as described previously (Gruening et al., 2006).

Real-time quantitative RT-PCR (qRT-PCR). Wild-type strain 10 and the $c c p A$-deficient mutant strain $10 \triangle c c p A$ were grown in THB medium to early exponential $\left(\mathrm{P}_{0}\right)$ and early stationary $\left(\mathrm{P}_{3}\right)$ phase, and RNA was prepared as described above. Two micrograms of RNA were reverse-transcribed with random primers (Promega) and analysed by 
real-time qRT-PCR with a Stratagene Mx3005P system (primers listed in Supplementary Table S1). The reaction mixture contained $2 \mu \mathrm{l}$ cDNA, $400 \mathrm{nM}$ primers and $10 \mu \mathrm{l}$ SYBR Green Mix (Qiagen) in a total volume of $20 \mu \mathrm{l}$. The PCR conditions were $95{ }^{\circ} \mathrm{C}$ for $10 \mathrm{~min}$, $95{ }^{\circ} \mathrm{C}$ for $20 \mathrm{~s}, 55{ }^{\circ} \mathrm{C}$ for $30 \mathrm{~s}$ and $72{ }^{\circ} \mathrm{C}$ for $20 \mathrm{~s}$, followed by a melting curve of the product as control. Data were normalized to a non-regulated housekeeping gene $(d n a H)$, and the relative transcript levels were calculated by the $\Delta \Delta C_{\mathrm{T}}$ method. Statistical analyses were performed by one-way analysis of variance (ANOVA) using Dunnett's adjustment to the non-regulated $\operatorname{argR}$ gene.

cDNA microarray analysis. RNA was extracted from wild-type strain 10 and the respective mutant strain $10 \Delta c c p A$ that were grown in THB to $\mathrm{OD}_{600} 0.3\left(\mathrm{P}_{0}\right)$. Following this, cDNA microarray analysis was performed as previously described (Fulde et al., 2011).

Electron microscopy. For morphological analysis of the capsule structure, samples of early exponential $\left(\mathrm{P}_{0}\right)$-grown bacteria were fixed according to the lysine-acetate-based formaldehyde/glutaraldehyde ruthenium red-osmium (LRR) fixation procedure, as described previously (Benga et al., 2004). In addition, the capsule thickness of randomly selected bacteria was measured by the iTEM software.

Neutrophil killing assay. Neutrophil killing in the presence of $20 \%$ $(\mathrm{v} / \mathrm{v})$ naive porcine serum was assayed as described by Baums et al. (2009), except for the following modifications. S. suis strains 10 , $10 \Delta c p s \mathrm{EF}, 10 \Delta c c p A$ and $c 10 \Delta c c p A$ were grown in THB to $\mathrm{P}_{0}$. Glycerol ( $15 \%$, v/v, final volume) was added to each culture, and $500 \mu \mathrm{l}$ aliquots were flash-frozen in liquid nitrogen. Frozen cultures were kept at $-80{ }^{\circ} \mathrm{C}$. The same batch of frozen cultures was used during the whole experiment. The bacteria were incubated with porcine neutrophils at an m.o.i. of $1: 1$, as described by Baums et al. (2009). Strain $10 \Delta c p s$ EF was included as a positive control in all killing assays. Results were expressed as survival factors, representing the ratio of c.f.u. at $1 \mathrm{~h}$ to c.f.u. at time 0 . Finally, the survival factor was divided by the bacterial growth rate of each strain in RPMI medium without neutrophils, resulting in the relative survival factor. Statistical analysis was performed using ANOVA followed by a post-Tukey test.

Porcine plasma protein binding assay. For detecting porcine plasma binding, strains $10,10 \Delta c c p A, 10 \Delta c p s E F$ and $c 10 \Delta c c p A$ were grown to the early exponential $\left(\mathrm{P}_{0}\right)$ growth phase, harvested and resuspended in $2 \mathrm{ml}$ PBS. Equal amounts were controlled by determination of c.f.u. $\mathrm{ml}^{-1}$. Bacterial suspensions were mixed with $5 \mathrm{ml}$ porcine plasma prepared from fresh porcine heparinized blood and incubated for $2 \mathrm{~h}$ at room temperature on a rotator. After centrifugation for $15 \mathrm{~min}$ at $2316 \mathrm{~g}$, the precipitated pellet was washed three times with $5 \mathrm{ml}$ PBS. Bound proteins were eluted by addition of $1 \mathrm{ml}$ glycine buffer $(0.1 \mathrm{M}, \mathrm{pH} 2.0)$ and incubated on a rotator for $10 \mathrm{~min}$ at room temperature. Then, samples were centrifuged for $7 \mathrm{~min}$ at $8000 \mathrm{~g}$, and supernatants were neutralized with $1 \mathrm{M}$ Tris/ $\mathrm{HCl}$ ( $\mathrm{pH} \mathrm{8.0)}$ and subsequently concentrated by Microcon (Millipore) ultrafiltration with a cut-off of $10 \mathrm{kDa}$. The concentrated proteins were separated on an SDS gel and either stained with Coomassie brilliant blue or transferred onto a PVDF membrane for soluble fibrinogen detection using an anti-human fibrinogen antibody (Sigma, diluted $1: 1000$ in $1 \%$ BSA).

Haemolytic activity assay. The haemolytic activity of streptococcal culture supernatants due to the secreted toxin suilysin was determined as described by Schaufuss et al. (2007), with some modifications. Briefly, strains $10,10 \Delta c c p A$ and $10 \Delta s l y$ were grown to the early exponential $\left(\mathrm{P}_{0}\right)$ growth phase and pelleted by centrifugation. A $100 \mu \mathrm{l}$ volume of serial twofold-diluted bacterial supernatants was mixed with $100 \mu 12 \%$ sheep red blood cells. The plates were incubated at $37{ }^{\circ} \mathrm{C}$ for $2 \mathrm{~h}$, and non-lysed erythrocytes were removed by centrifugation at $1000 \mathrm{~g}$ for $5 \mathrm{~min}$. A $100 \mu \mathrm{l}$ volume of the supernatant was transferred to a flat-bottomed, 96-well microtitre plate for determination of $A_{550}$ (Tecan, GENios Pro reader). Results were expressed as percentage haemolysis (haemolytic activity) compared with hypo-osmotic haemolysis by double-distilled $\mathrm{H}_{2} \mathrm{O}$.

\section{RESULTS}

\section{Growth-dependent expression of virulence- associated factors}

Many factors have been shown to contribute to S. suis virulence, but for only a few of them has gene regulation been elucidated. One example for an environmentally highly regulated virulence-associated factor is the ADS. As an alternative energy-providing system, the ADS has been shown to be subject to CCR (Gruening et al., 2006). To analyse glucose-mediated regulation of virulence, we first related glucose availability during $S$. suis growth to ADS expression, to characterize more precisely the growth phases with high and low glucose. ArcB expression was used to indicate ADS expression during growth of $S$. suis (Fig. 1a). ArcB expression was inversely related to glucose concentration; for example, ArcB expression was highest under glucose-deprivation conditions, i.e. in the early stationary growth phase $\left(\mathrm{P}_{3}\right)$. Based on these findings, we next compared the expression of eight selected well-known virulence-associated genes, including $\operatorname{arcB}$, for $\operatorname{arc} A B C$ operon expression, cps $2 A$, representing capsule locus expression, sly, ofs, sao, eno, $\mathrm{mrp}$ and epf, between early exponential $\left(\mathrm{P}_{0}\right.$, high glucose $)$ and early stationary $\left(\mathrm{P}_{3}\right.$, low glucose) growth. As shown in Fig. 1(b), with the exception of eno and $m r p$, all other genes showed growth phasedependent differential expression. Expression of $\operatorname{arcB}$ and sly was highly upregulated in stationary phase-grown bacteria, whereas the relative transcript levels of $\operatorname{cps} 2 \mathrm{~A}$ and the surface-located proteins sao and ofs were lower as compared with the early exponential growth phase. These results suggested a glucose-mediated regulation of virulence-associated factors.

\section{Effect of $\operatorname{ccpA}$ depletion on virulence-associated factor expression}

CCR in Gram-positive bacteria is mediated by the transcriptional regulator CcpA. To analyse the role of CcpA in growth-dependent virulence gene expression, we constructed the $c c p A$-deficient strain $10 \Delta c c p A$ (Supplementary Fig. S1). Insertion of the antibioticresistance cassette did not affect expression of the genes located up- and downstream of ccpA (SSU1202; Supplementary Table S2). The growth of the CcpAdeficient strain was similar to that of the wild-type strain 10 (Fig. 2a). However, as expected, CCR of $\operatorname{arc} A B C$ operon expression was abolished, indicated by the elevated $\mathrm{ArcB}$ expression during early exponential growth $\left(\mathrm{P}_{0}\right)$ in strain $10 \triangle c c p A$ (Fig. 2b). Next, we analysed virulence-associated factor regulation in the $c c p A$-knockout strain. For this, we related the relative transcript levels of $\operatorname{arcB}, s l y$, ofs, sao, eno, 
$c p s 2 A, m r p$ and epf in wild-type strain 10 and strain $10 \triangle c c p A$ during early exponential growth, when glucose is still available (Fig. 2c). Corresponding to the expression of the ADS in the absence of CcpA (Fig. 2b), arcB mRNA levels were significantly higher in the mutant strain. However, mRNA levels of nearly all other genes were reduced after CcpA depletion. Thus, during early exponential growth, ofs, sao, eno and cps $2 A$ transcript levels were significantly lower in the mutant strain. The amounts of sly and $m r p$ mRNA were also reduced, and only expression of epf was similar in the wild-type strain and the mutant. Together, these data show that CcpA depletion both positively and negatively influences the expression of virulence-associated factors.

\section{Effect of $\operatorname{ccpA}$ depletion on global gene expression}

To gain more insight into the role of CcpA in S. suis virulence and metabolism, we performed whole-genome cDNA microarray analyses with RNA extracted from strain $10 \triangle c c p A$ and wild-type strain 10 during early exponential growth $\left(\mathrm{P}_{0}\right)$. The comparison of microarray data revealed 259 differentially expressed genes $(13.2 \%$ of the $S$. suis genome) with expression changes of greater than twofold. The expression of 141 genes was higher, and 118 genes showed lower expression in $10 \Delta c c p A$ (Supplementary Table S2). As shown by immunoblot analysis, the $\operatorname{arc} A B C$ operon ( $>11$-fold higher expression) belonged to the most strongly CcpA-repressed genes during early exponential growth. A Clusters of Orthologous Groups (COG) analysis of all affected genes is shown in Table 1. The majority of differentially expressed genes (56) in strain $10 \Delta c c p A$ were related to carbohydrate transport. Notably, genes associated with cell wall biogenesis, including the entire capsule synthesis $(c p s)$ and sialic acid synthesis (neu) gene clusters, were strongly affected by the $\operatorname{ccp} A$ knockout. Similar to other Grampositive bacteria, including $S$. pyogenes (Kinkel \& McIver, 2008; Moreno et al., 2001; Shelburne et al., 2008; Zomer et al., 2007), in S. suis, CcpA seems to negatively regulate several operons for sugar utilization in the exponential growth phase. In detail, a maltose/maltodextrin $A B C$ transporter and putative sugar-specific phosphotransferase system (PTS) for maltose, mannose, fructose, lactose and $\mathrm{N}$-acetylgalactosamine were upregulated in strain $10 \triangle c c p A$. The $c c p A$ knockout positively and negatively influenced the mRNA level of 14 other transcriptional regulators. Interestingly, as CcpA has mainly been described as a repressor, the $c c p A$ knockout resulted in a downregulation of about $6 \%$ of all ORFs throughout the genome. Besides the capsule synthesis cluster (cps2ABCEDFGHIJ), we found other genes encoding virulence-associated factors, such as opacity factor $(o f s)$, surface-anchored SAO protein (sao) and enolase (eno), to be negatively affected by the $c c p A$ knockout, which corresponded to our qRT-PCR shown in Fig. 2(c).
Table 1. Comparative microarray analysis of wild-type strain 10 and strain $10 \Delta c c p A$ grown to early exponential phase $\left(P_{0}\right)$

\begin{tabular}{|c|c|c|}
\hline Putative function ${ }^{\star}$ & $\begin{array}{c}\text { Genes with } \\
\text { lower expression } \\
\text { in strain } \\
10 \Delta c c p A\end{array}$ & $\begin{array}{c}\text { Genes with } \\
\text { higher } \\
\text { expression in } \\
\text { strain } 10 \Delta c c p A\end{array}$ \\
\hline Energy production (C) & 6 & 6 \\
\hline Cell cycle control (D) & - & 1 \\
\hline Amino acid transport (E) & 8 & 9 \\
\hline Nucleotide transport (F) & 1 & 9 \\
\hline Carbohydrate transport $(\mathrm{G})$ & 11 & 45 \\
\hline Coenzyme transport $(\mathrm{H})$ & - & 1 \\
\hline Lipid transport (I) & 6 & 1 \\
\hline Translation (J) & - & 4 \\
\hline Transcription (K) & 7 & 7 \\
\hline Replication (L) & - & 1 \\
\hline Cell wall biogenesis (M) & 18 & - \\
\hline $\begin{array}{l}\text { Post-translational } \\
\text { modification }(\mathrm{O})\end{array}$ & 6 & 3 \\
\hline Inorganic ion transport $(\mathrm{P})$ & 3 & 7 \\
\hline General function prediction (R) & 3 & 16 \\
\hline Function unknown $(\mathrm{S})$ & 8 & 5 \\
\hline Signal transduction $(\mathrm{T})$ & 2 & 1 \\
\hline Defence mechanism (V) & 3 & - \\
\hline Virulence-associated (VAF) & 2 & - \\
\hline Hypothetical proteins $(-)$ & 34 & 25 \\
\hline Sum & 118 & 141 \\
\hline $\begin{array}{l}\text { Total (percentage of whole } \\
\text { genome) }\end{array}$ & $259(13.2 \%)$ & \\
\hline
\end{tabular}

${ }^{\star} \mathrm{COG}$ analysis was performed for differentially expressed genes and the respective one-letter code is shown in parentheses.

\section{Effect of ccpA depletion on capsule-associated virulence properties}

The virulence of $S$. suis is closely connected to capsule expression, at least in serotype 2 strains. As demonstrated above, the transcript levels of all genes contributing to capsule expression as well as several other genes contributing to cell wall synthesis were reduced in strain $10 \Delta c c p A$, suggesting that CcpA depletion alters capsule and cell wall structure. Therefore, we studied the capsules of wild-type strain 10 , the capsular mutant $10 \Delta c p s E F$, strain $10 \Delta c c p A$ and the complemented strain $c 10 \Delta c c p A$ during early exponential growth $\left(\mathrm{P}_{0}\right)$ by electron microscopy. As shown in Fig. 3, capsule expression was substantially reduced in strain $10 \Delta c c p A$. The wild-type phenotype could be restored after complementation of strain $10 \Delta c c p A$ with plasmidencoded CcpA. Quantification of capsule thickness revealed that compared with the wild-type strain 10 , the capsule thickness of strain $10 \triangle c c p A$ was significantly reduced (Fig. 4a). Thus, the CcpA-deficient phenotype closely resembled that of the capsular mutant $10 \Delta c p s E F$. In the latter, however, capsule expression was completely abolished. The wild-type phenotype could be restored 

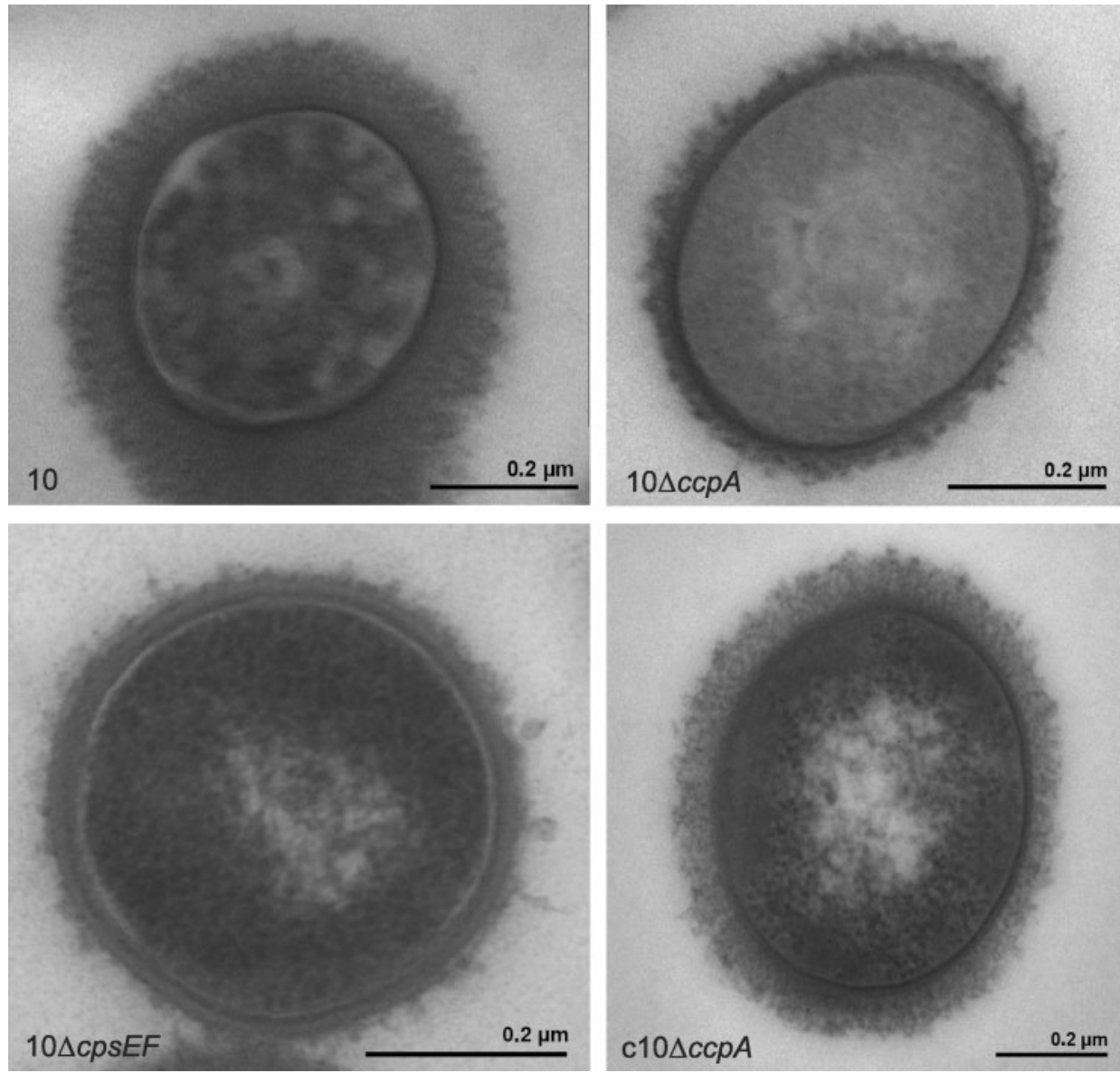

Fig. 3. Effect of $\operatorname{ccpA}$ depletion on bacterial encapsulation. Characterization of capsule expression of $S$. suis strains by LRR staining and transmission electron microscopy. Electron microscopy pictures of $S$. suis wild-type strain 10, strain $10 \Delta c c p A$, strain $10 \Delta c p s E F$ and strain $c 10 \Delta c c p A$ grown in THB medium to early exponential phase $\left(\mathrm{P}_{0}\right)$.

after complementation of strain $10 \Delta c c p A$ with plasmidencoded CcpA $(\operatorname{c10} \Delta c c p A)$.

The above data indicated that CcpA depletion affected capsule expression. Therefore, we tested the binding capacity of bacteria to porcine plasma proteins. Fig. 4(b) shows a Coomassie brilliant blue-stained SDS gel of eluted bacteria-bound plasma proteins. Strain $10 \Delta c p s E F$ possessed the highest binding capacity to porcine plasma proteins compared with the encapsulated wild-type strain 10. Strain $10 \Delta c c p A$ displayed a higher binding than that of wild-type strain 10 but lower than that of strain $10 \Delta c p s E F$. Correspondingly, using Western blot analysis, we found binding of porcine fibrinogen to strains $10 \Delta c c p A$ and $10 \Delta c p s E F$, but not to the wild-type strain 10. Notably, binding capacity was abolished by complementation of strain $10 \Delta c c p A$ with plasmid-encoded CcpA (c10 $\Delta c c p A)$. Next, we studied the ability of the CcpA mutant strain to resist killing by porcine polymorphonuclear leukocytes (PMNs). For this, wild-type strain 10 and mutant strain $10 \Delta c c p A$ were incubated with porcine PMNs in the presence of naive swine sera. As shown in Fig. 4(c), the relative survival of strain $10 \Delta c c p A$ was significantly reduced ( $\sim 62$ vs $\sim 100 \%$ ) compared with the wild-type strain 10. The non-encapsulated strain $10 \Delta c p s E F$, which we used as a control, was not able to resist phagocytic killing. The effects were not due to cytotoxicity, as the haemolytic activities of wild-type strain 10 and the mutant strain $10 \triangle c c p A$ were similar (Fig. 4d). Complementation of the CcpA knock-out strain restored killing to the wildtype level. These results indicated that the CcpA depletion was responsible for the reduced resistance of $S$. suis to phagocytic killing.

\section{DISCUSSION}

It is well accepted that nutrient availability in different ecological niches of the host directs the expression of the virulence features of pathogenic bacteria. One prominent example of this is capsule expression during pneumococcal infection (Kadioglu et al., 2008). Thus, levels of $c p s$ mRNA 
(a)

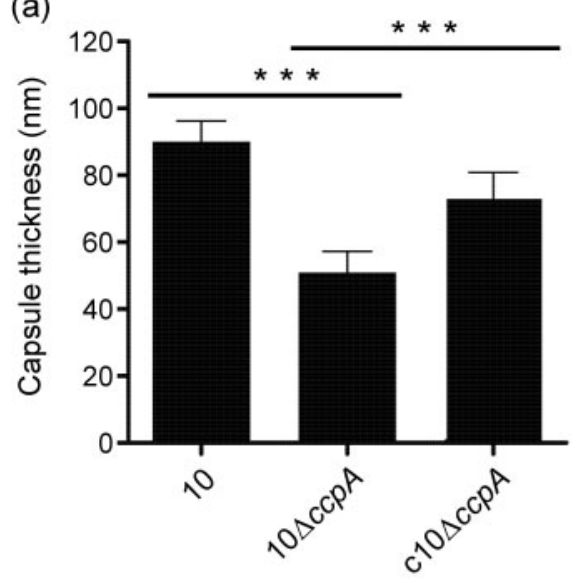

(b)

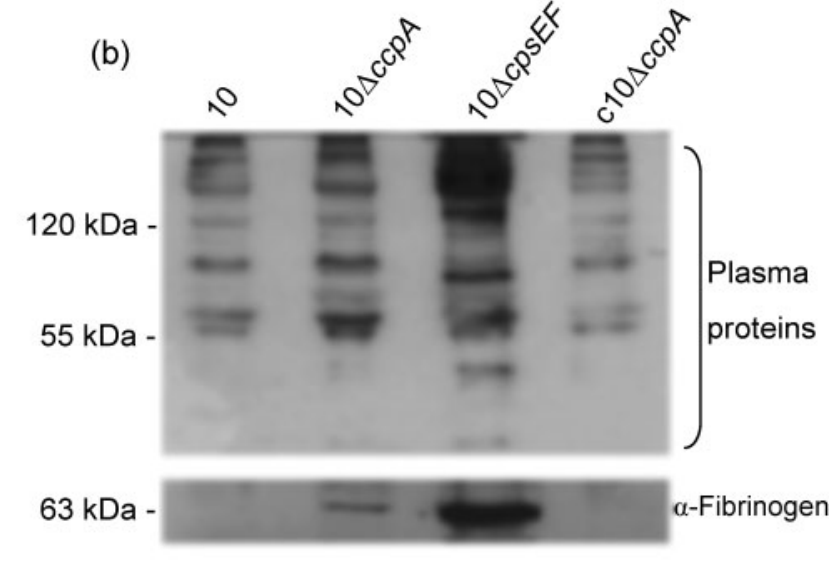

(d)

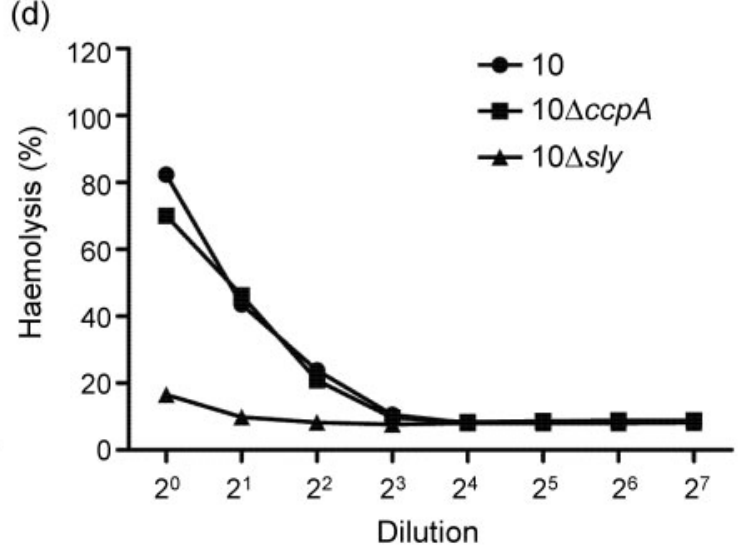

Fig. 4. Effect of $c c p A$ depletion on bacterial virulence properties. (a) Capsule thickness of early exponential-grown strains 10 , $10 \Delta c c p A$ and $c 10 \Delta c c p A$, as determined by electron microscopy based on measurement of 30 randomly selected bacteria with the iTEM software. ${ }^{* *}$ Significance of an unpaired $t$ test for $P<0.001$. (b) Porcine plasma binding of $S$. suis. Plasma binding was performed as described in Methods. Porcine plasma proteins bound by early exponential-grown bacteria were visualized by SDSPAGE and Coomassie staining (upper panel). Bound proteins were blotted onto a PVDF membrane, and binding of bacterial strains to porcine fibrinogen is shown by Western blotting with an anti-fibrinogen antibody (lower panel). (c) Survival of $S$. suis wild-type strain 10, strain $10 \Delta c c p A$, strain $10 \Delta c p s E F$ and strain $c 10 \Delta c c p A$ in the presence of porcine neutrophils and piglet naive serum. Bacteria were grown in THB medium to early exponential phase $\left(P_{0}\right)$, and a bacterial survival assay was performed as described in Methods, including statistics. Data are mean \pm SD of three biological replicates. The significance of an unpaired $t$ test is indicated ( ${ }^{\star} P<0.05,{ }^{\star \star} P<0.01$ ). (d) Haemolytic activities of $S$. suis culture supernatants of wild-type strain 10, strain $10 \Delta c c p A$ and strain $10 \Delta s$ s. Bacteria were grown to the early exponential $\left(P_{0}\right)$ growth phase. Culture supernatants were twofold-diluted and erythrocyte lysis was monitored by measurement of $\mathrm{OD}_{550}$. Results are given for one representative experiment.

are significantly different between pneumococci isolated from the blood of infected mice and those grown in vitro (Ogunniyi et al., 2002). At present, there is only indirect evidence that capsule expression in S. pneumoniae (and some other pathogenic streptococci) is regulated by glucose availability via RegM/CcpA (Giammarinaro \& Paton, 2002). In the present study, we found that glucose mediates virulence-associated gene expression via CcpA in S. suis. Furthermore, we demonstrate for what is believed to be the first time that CcpA is necessary for capsule expression and, thereby, is important for resistance to phagocytosis in S. suis.
CcpA has long been studied as a global bacterial sensor of glucose availability. More recently, it has become clear that such metabolic regulators can also contribute to the virulence of pathogens (Hondorp \& McIver, 2007; Poncet et al., 2009). As a global regulator, CcpA has been investigated in several Gram-positive bacteria, including Bacillus subtilis, Lactococcus lactis and some streptococci (Deutscher, 2008; Sonenshein, 2007; Zomer et al., 2007).

Since growth phase-dependent regulation of virulence factor expression can influence pathogen fitness within 
the host (Kreikemeyer et al., 2003), we analysed the expression of virulence-associated factors during growth to reflect the adaptation of a pathogen to changing host environments (Hondorp \& McIver, 2007; Mekalanos, 1992). Thus, in group A streptococci (GAS), a correlation between in vivo expression patterns and in vitro cultures of early stationary-grown bacteria has been observed (Cho \& Caparon, 2005). Here, we found that the expression of major virulence-associated factors of $S$. suis, including $\operatorname{arc} A B C$, sly, ofs, sao, cps $2 A$ and $e p f$, is dependent on the bacterial growth phase. The $\operatorname{arc} A B C$ operon is controlled by CCR (Gruening et al., 2006; Zeng et al., 2006). As a consequence, it was strongly induced when the glucose concentration in the medium decreased. Since CcpA has been described as the main regulatory sensor of CCR, we constructed a $c c p A$-knockout strain. Deletion mutagenesis resulted in the abolition of the repressive glucose effect and an uncoupling of $\operatorname{arc} A B C$ expression from growth phase regulation. Based on this observation, CCR mediated by putative orthologous pathways such as CcpB (Chauvaux et al., 1998), CcpC (Jourlin-Castelli et al., 2000), CcpN (Eckart et al., 2009) and the PTS (Deutscher, 2008) could be excluded for arcABC expression control. Overall, these observations fit well with results observed for other streptococci (Chaussee et al., 2003; Dong et al., 2004; Shelburne et al., 2008).

To determine the possible role of CcpA in the regulation of other virulence-associated factors we compared virulence gene expression of the wild-type strain 10 with that of strain $10 \Delta c c p A$. During exponential growth (Fig. 2c), the relative expression levels of $\operatorname{arcB}, \operatorname{cps} 2 A$, ofs and sao showed the same regulation pattern in exponential and stationary growth in the wild-type strain. These observations led us to presume that CcpA was the mediator of the growth phasedependent regulation of these virulence-associated factors. Analysing the global expression differences in strain $10 \triangle c c p A$ revealed 259 differentially expressed genes in early exponential growth when the glucose levels in the growth medium were still high. CcpA deficiency resulted in $118(45.5 \%)$ down- and 141 (54.5\%) upregulated genes, which differ in the relative distribution from what has been published for GAS in which approximately $90 \%$ of the differentially regulated genes between the wild-type and a $c с p A$ knockout have been found to be repressed by CcpA (Kinkel \& McIver, 2008; Shelburne et al., 2008).

Four genes were confirmed by real-time qRT-PCR, indicating a significant correlation of the assays (Supplementary Fig. S2). COG analysis revealed that 56 genes (45 upregulated, 11 downregulated) of the 259 affected genes in the CcpA-knockout strain were related to carbohydrate transport and 17 (nine upregulated, eight downregulated) to amino acid transport, as well as 12 (six upregulated, six downregulated) to energy production, indicating the relevance of CcpA as a metabolic regulator in S. suis. Thus, the microarray data confirmed the repressive effect of CcpA on the $\operatorname{arcABC}$ operon, supporting earlier studies in other streptococci (Chaussee et al.,
2003; Dong et al., 2004). CcpA depletion relieved CCR of operons for carbohydrate acquisition, such as the mannose-specific PTS (manLMN), $N$-acetylgalactosamine PTS (agaVWD), fructose-specific PTS ( fruA), cellobiose/lactose PTS (SSU1855-SSU1859), a maltose/maltodextrin-binding protein (malX) and an ABC-transport system (malCDAR), as well as the glycogen synthase pathway $(g l g C A B)$, which has been postulated to be repressed by CcpA in B. subtilis (Deutscher et al., 2006).

Among the differentially expressed genes we found 14 transcriptional regulators (seven upregulated, seven downregulated), further explaining the impact of the ccpA knockout on the whole regulatory network of $S$. suis transcription. Another very striking observation was that 18 genes were relieved from CCA, i.e. their expression was considerably lower in the mutant strain. The majority of these genes are responsible for the synthesis of the capsule, the most important virulence factor of S. suis. The capsule of S. suis serotype 2 strains is composed of galactose, glucose, $\mathrm{N}$-acetyl-D-glucosamine, rhamnose and $\mathrm{N}$-acetylneuraminic acid (sialic acid) (Elliott \& Tai, 1978; Van Calsteren et al., 2010). In the microarray the entire capsule synthesis cluster cps $2 A B C E D F G H I J$ and, additionally, the sialic acid synthase cluster (neuBCDA) were downregulated in strain $10 \Delta c c p A$. This finding has also been observed in other streptococci, including S. pneumoniae (Giammarinaro \& Paton, 2002; Shelburne et al., 2008), but none of these studies analysed it more in depth.

We focused on the phenotypic characterization of strain $10 \Delta c c p A$, with emphasis on capsule-associated features under glucose-rich conditions. Electron microscopy demonstrated that the capsule thickness was considerably reduced in strain $10 \Delta c c p A$. Complementation of the mutant strain restored capsule thickness to the wild-type level, confirming the importance of CcpA for capsule expression.

Capsule expression of S. suis is of particular importance for resistance to uptake and killing by mononuclear phagocytes (Segura et al., 2004; Smith et al., 1999). It is hypothesized that during infection, S. suis first downregulates capsule expression for increased adhesion to epithelial cells and then upregulates it for protection against phagocytosis after entering the bloodstream (Gottschalk \& Segura, 2000). Accordingly, the increased plasma protein-binding capacity of strain $10 \Delta c c p A$ (Fig. $4 \mathrm{~b}$ ) indicated a more adhesive phenotype, which would be necessary for adhesion (and invasion) in a low-glucose environment. However, despite the reduced thickness of the capsule in strain $10 \Delta c c p A$, biofilm formation and adherence to epithelial cells were not altered (data not shown). A possible explanation is that CcpA deficiency might affect the expression of other components necessary for biofilm formation and/or not-yet-characterized adhesins. Thus, the lower expression of the surface-located virulence-associated factors SAO, OFS and enolase may contribute to the observed phenotype, though their role in 
adhesion has not yet been proven. On the other hand, the resistance of strain $10 \Delta c c p A$ to neutrophil killing was markedly reduced, indicating that CcpA is necessary for capsule expression of $S$. suis in glucose-rich environments. This might be of relevance, for example when $S$. suis enters the bloodstream of the host, where glucose is the major sugar source (5.4 mM) (Baker et al., 2007), in order to facilitate resistance to killing by porcine neutrophils. Nevertheless, the lower expression of known surfacelocated proteins (SAO, OFS and enolase) and yetuncharacterized proteins may also contribute to resistance to phagocytic killing.

In conclusion, our results show the relevance of the global regulator CcpA for virulence-associated factor expression in S. suis, and underline the link between metabolism and virulence in bacterial pathogens.

\section{ACKNOWLEDGEMENTS}

We gratefully acknowledge Christoph Baums for providing plasmid $p \mathrm{IC}_{\mathrm{erm}}$ and for contributing to our plasma protein-binding studies. We thank the Klinik für Klauentiere of the Stiftung Tierärztliche Hochschule Hannover for providing porcine blood. This work was supported by the Deutsche Forschungsgemeinschaft (DFG), Bonn, Germany (SFB 578), and a Georg Christoph Lichtenberg stipend to J.W. [Zentrum für Infektionsbiologie; ZIB (Center for Infection Biology)].

\section{REFERENCES}

Arends, J. P. \& Zanen, H. C. (1988). Meningitis caused by Streptococcus suis in humans. Rev Infect Dis 10, 131-137.

Baker, E. H., Clark, N., Brennan, A. L., Fisher, D. A., Gyi, K. M., Hodson, M. E., Philips, B. J., Baines, D. L. \& Wood, D. M. (2007). Hyperglycemia and cystic fibrosis alter respiratory fluid glucose concentrations estimated by breath condensate analysis. J Appl Physiol 102, 1969-1975.

Baums, C. G. \& Valentin-Weigand, P. (2009). Surface-associated and secreted factors of Streptococcus suis in epidemiology, pathogenesis and vaccine development. Anim Health Res Rev 10, 65-83.

Baums, C. G., Kaim, U., Fulde, M., Ramachandran, G., Goethe, R. \& Valentin-Weigand, P. (2006). Identification of a novel virulence determinant with serum opacification activity in Streptococcus suis. Infect Immun 74, 6154-6162.

Baums, C. G., Kock, C., Beineke, A., Bennecke, K., Goethe, R., Schröder, C., Waldmann, K. H. \& Valentin-Weigand, P. (2009). Streptococcus suis bacterin and subunit vaccine immunogenicities and protective efficacies against serotypes 2 and 9. Clin Vaccine Immunol 16, 200-208.

Benga, L., Goethe, R., Rohde, M. \& Valentin-Weigand, P. (2004). Nonencapsulated strains reveal novel insights in invasion and survival of Streptococcus suis in epithelial cells. Cell Microbiol 6, 867-881.

Benga, L., Fulde, M., Neis, C., Goethe, R. \& Valentin-Weigand, P. (2008). Polysaccharide capsule and suilysin contribute to extracellular survival of Streptococcus suis co-cultivated with primary porcine phagocytes. Vet Microbiol 132, 211-219.

Chanter, N., Jones, P. W. \& Alexander, T. J. (1993). Meningitis in pigs caused by Streptococcus suis - a speculative review. Vet Microbiol 36, $39-55$.
Charland, N., Harel, J., Kobisch, M., Lacasse, S. \& Gottschalk, M. (1998). Streptococcus suis serotype 2 mutants deficient in capsular expression. Microbiology 144, 325-332.

Chaussee, M. S., Somerville, G. A., Reitzer, L. \& Musser, J. M. (2003). Rgg coordinates virulence factor synthesis and metabolism in Streptococcus pyogenes. J Bacteriol 185, 6016-6024.

Chauvaux, S., Paulsen, I. T. \& Saier, M. H., Jr (1998). CcpB, a novel transcription factor implicated in catabolite repression in Bacillus subtilis. J Bacteriol 180, 491-497.

Cho, K. H. \& Caparon, M. G. (2005). Patterns of virulence gene expression differ between biofilm and tissue communities of Streptococcus pyogenes. Mol Microbiol 57, 1545-1556.

Clifton-Hadley, F. A. \& Alexander, T. J. (1980). The carrier site and carrier rate of Streptococcus suis type II in pigs. Vet Rec 107, 40-41.

Deutscher, J. (2008). The mechanisms of carbon catabolite repression in bacteria. Curr Opin Microbiol 11, 87-93.

Deutscher, J., Francke, C. \& Postma, P. W. (2006). How phosphotransferase system-related protein phosphorylation regulates carbohydrate metabolism in bacteria. Microbiol Mol Biol Rev 70, 9391031.

Dong, Y. Q., Chen, Y. Y. M. \& Burne, R. A. (2004). Control of expression of the arginine deiminase operon of Streptococcus gordonii by CcpA and Flp. J Bacteriol 186, 2511-2514.

Eckart, R. A., Brantl, S. \& Licht, A. (2009). Search for additional targets of the transcriptional regulator $\mathrm{CcpN}$ from Bacillus subtilis. FEMS Microbiol Lett 299, 223-231.

Elliott, S. D. \& Tai, J. Y. (1978). The type-specific polysaccharides of Streptococcus suis. J Exp Med 148, 1699-1704.

Fulde, M., Willenborg, J., de Greeff, A., Benga, L., Smith, H., ValentinWeigand, P. \& Goethe, R. (2011). ArgR is an essential local transcriptional regulator of the $\operatorname{arc} A B C$ operon in Streptococcus suis and is crucial for biological fitness in an acidic environment. Microbiology 157, 572-582.

Giammarinaro, P. \& Paton, J. C. (2002). Role of RegM, a homologue of the catabolite repressor protein CcpA, in the virulence of Streptococcus pneumoniae. Infect Immun 70, 5454-5461.

Goethe, R. \& Phi-van, L. (1998). Posttranscriptional lipopolysaccharide regulation of the lysozyme gene at processing of the primary transcript in myelomonocytic HD11 cells. J Immunol 160, 4970-4978.

Gottschalk, M. \& Segura, M. (2000). The pathogenesis of the meningitis caused by Streptococcus suis: the unresolved questions. Vet Microbiol 76, 259-272.

Gottschalk, M., Xu, J., Calzas, C. \& Segura, M. (2010). Streptococcus suis: a new emerging or an old neglected zoonotic pathogen? Future Microbiol 5, 371-391.

Gruening, P., Fulde, M., Valentin-Weigand, P. \& Goethe, R. (2006). Structure, regulation, and putative function of the arginine deiminase system of Streptococcus suis. J Bacteriol 188, 361-369.

Hondorp, E. R. \& Mclver, K. S. (2007). The Mga virulence regulon: infection where the grass is greener. Mol Microbiol 66, 1056-1065.

Iyer, R., Baliga, N. S. \& Camilli, A. (2005). Catabolite control protein A (CcpA) contributes to virulence and regulation of sugar metabolism in Streptococcus pneumoniae. J Bacteriol 187, 8340-8349.

Jourlin-Castelli, C., Mani, N., Nakano, M. M. \& Sonenshein, A. L. (2000). CcpC, a novel regulator of the LysR family required for glucose repression of the citB gene in Bacillus subtilis. J Mol Biol 295, 865-878.

Kadioglu, A., Weiser, J. N., Paton, J. C. \& Andrew, P. W. (2008). The role of Streptococcus pneumoniae virulence factors in host respiratory colonization and disease. Nat Rev Microbiol 6, 288-301. 
Kietzman, C. C. \& Caparon, M. G. (2010). CcpA and LacD.1 affect temporal regulation of Streptococcus pyogenes virulence genes. Infect Immun 78, 241-252.

Kinkel, T. L. \& Mclver, K. S. (2008). CcpA-mediated repression of streptolysin $\mathrm{S}$ expression and virulence in the group A streptococcus. Infect Immun 76, 3451-3463.

Kreikemeyer, B., Mclver, K. S. \& Podbielski, A. (2003). Virulence factor regulation and regulatory networks in Streptococcus pyogenes and their impact on pathogen-host interactions. Trends Microbiol 11, 224232.

Mekalanos, J. J. (1992). Environmental signals controlling expression of virulence determinants in bacteria. J Bacteriol 174, 1-7.

Moreno, M. S., Schneider, B. L., Maile, R. R., Weyler, W. \& Saier, M. H., Jr (2001). Catabolite repression mediated by the CcpA protein in Bacillus subtilis: novel modes of regulation revealed by whole-genome analyses. Mol Microbiol 39, 1366-1381.

Ogunniyi, A. D., Giammarinaro, P. \& Paton, J. C. (2002). The genes encoding virulence-associated proteins and the capsule of Streptococcus pneumoniae are upregulated and differentially expressed in vivo. Microbiology 148, 2045-2053.

Poncet, S., Milohanic, E., Mazé, A., Nait Abdallah, J., Aké, F., Larribe, M., Deghmane, A. E., Taha, M. K., Dozot, M. \& other authors (2009). Correlations between carbon metabolism and virulence in bacteria. Contrib Microbiol 16, 88-102.

Rosenkranz, M., Elsner, H. A., Stürenburg, H. J., Weiller, C., Röther, J. \& Sobottka, I. (2003). Streptococcus suis meningitis and septicemia contracted from a wild boar in Germany. J Neurol 250, 869-870.

Sambrook, J., Fritsch, E. F. \& Maniatis, T. (1989). Molecular Cloning: a Laboratory Manual, 2nd edn. Cold Spring Harbor, NY: Cold Spring Harbor Laboratory.

Schaufuss, P., Müller, F. \& Valentin-Weigand, P. (2007). Isolation and characterization of a haemolysin from Trichophyton mentagrophytes. Vet Microbiol 122, 342-349.

Segura, M., Gottschalk, M. \& Olivier, M. (2004). Encapsulated Streptococcus suis inhibits activation of signaling pathways involved in phagocytosis. Infect Immun 72, 5322-5330.

Seshasayee, A. S. N., Bertone, P., Fraser, G. M. \& Luscombe, N. M. (2006). Transcriptional regulatory networks in bacteria: from input signals to output responses. Curr Opin Microbiol 9, 511-519.

Shelburne, S. A., III, Keith, D., Horstmann, N., Sumby, P., Davenport, M. T., Graviss, E. A., Brennan, R. G. \& Musser, J. M. (2008). A direct link between carbohydrate utilization and virulence in the major human pathogen group A Streptococcus. Proc Natl Acad Sci U S A 105, $1698-1703$.
Smith, H. E., Wisselink, H. J., Vecht, U., Gielkens, A. L. J. \& Smits, M. A. (1995). High-efficiency transformation and gene inactivation in Streptococcus suis type 2. Microbiology 141, 181-188.

Smith, H. E., Damman, M., van der Velde, J., Wagenaar, F., Wisselink, H. J., Stockhofe-Zurwieden, N. \& Smits, M. A. (1999). Identification and characterization of the cps locus of Streptococcus suis serotype 2: the capsule protects against phagocytosis and is an important virulence factor. Infect Immun 67, 1750-1756.

Sonenshein, A. L. (2007). Control of key metabolic intersections in Bacillus subtilis. Nat Rev Microbiol 5, 917-927.

Titgemeyer, F. \& Hillen, W. (2002). Global control of sugar metabolism: a Gram-positive solution. Antonie van Leeuwenhoek 82, 59-71.

Van Calsteren, M. R., Gagnon, F., Lacouture, S., Fittipaldi, N. \& Gottschalk, M. (2010). Structure determination of Streptococcus suis serotype 2 capsular polysaccharide. Biochem Cell Biol 88, 513-525.

Vecht, U., Wisselink, H. J., van Dijk, J. E. \& Smith, H. E. (1992). Virulence of Streptococcus suis type 2 strains in newborn germfree pigs depends on phenotype. Infect Immun 60, 550-556.

Vecht, U., Wisselink, H. J., Stockhofe-Zurwieden, N. \& Smith, H. E. (1996). Characterization of virulence of the Streptococcus suis serotype 2 reference strain Henrichsen S 735 in newborn gnotobiotic pigs. Vet Microbiol 51, 125-136.

Wen, Z. T. \& Burne, R. A. (2002). Functional genomics approach to identifying genes required for biofilm development by Streptococcus mutans. Appl Environ Microbiol 68, 1196-1203.

Winterhoff, N., Goethe, R., Gruening, P., Rohde, M., Kalisz, H., Smith, H. E. \& Valentin-Weigand, P. (2002). Identification and characterization of two temperature-induced surface-associated proteins of Streptococcus suis with high homologies to members of the arginine deiminase system of Streptococcus pyogenes. J Bacteriol 184, 6768-6776.

Wisselink, H. J., Smith, H. E., Stockhofe-Zurwieden, N., Peperkamp, K. \& Vecht, U. (2000). Distribution of capsular types and production of muramidase-released protein (MRP) and extracellular factor (EF) of Streptococcus suis strains isolated from diseased pigs in seven European countries. Vet Microbiol 74, 237-248.

Zeng, L., Dong, Y. \& Burne, R. A. (2006). Characterization of cisacting sites controlling arginine deiminase gene expression in Streptococcus gordonii. J Bacteriol 188, 941-949.

Zomer, A. L., Buist, G., Larsen, R., Kok, J. \& Kuipers, O. P. (2007). Time-resolved determination of the CcpA regulon of Lactococcus lactis subsp. cremoris MG1363. J Bacteriol 189, 1366-1381.

Edited by: H. Ingmer 